\title{
VAC_06 - Stability evaluation of a reconstitute yellow fever vaccine and its application as reference material for potency assay
}

Ana Carolina Ferreira Ballestê Ajorio ${ }^{1 *}$; Anderson Peclat Rodrigues ${ }^{1}$; Vinícius Pessanha Rhodes ${ }^{1}$; Vanessa Alvaro Diniz ${ }^{1}$; Renata de Paula Souza ${ }^{1}$; Igor Barbosa da Silva ${ }^{1}$; Marcelo Luiz Lima Brandão ${ }^{1}$.

${ }^{1}$ Fiocruz/Bio-Manguinhos.

Introduction: The attenuated yellow fever vaccine (YFV) is offered free of charge to the Brazilian population through the National Immunization Program. One of the specifications for quality control release of the vaccine is the potency determination. This test consists in the determination of the number of plaque forming units (PFU), in which an established reference vaccine is used as a reference material (RM) to validate the results. Actually, the RM used is multidose (five doses) and after its reconstitution, one dose $(0.5 \mathrm{~mL})$ is used in the assay and the remaining four doses are discarded. So, stability studies are necessary in order to verify if this RM can be stored and used after its reconstitution.

Objective: The aim of this study was to evaluate the stability of one YFV batch produced by BioManguinhos, to be used as RM, after its reconstitution for the potency assay.

Methodology: The stability of the reconstitute $\mathrm{RM}$ was evaluated in short term at $(5 \pm 3)^{\circ} \mathrm{C}$ for three days using classic and isochronal model and at $(-20 \pm 10)^{\circ} \mathrm{C}$ for eight days using classic model. In the $(5 \pm 3)$ ${ }^{\circ} \mathrm{C}$ stability study, two vials were analyzed in each assay period of time (T0, T1, T2, and T3) in both models, totalizing 16 vials. In the $(-20 \pm 10)^{\circ} \mathrm{C}$ stability study, four vials were reconstituted and two pools (two vials/pool) were prepared and analyzed independently at $\mathrm{T} 0$, and the remain doses were distributed in 14 aliquots of $0.6 \mathrm{~mL}$ and stored at $(-20 \pm 10)^{\circ} \mathrm{C}$. In each period of time (T2, T3, T4, T5, T6, T7, and T8) two aliquots (one for each pool) were defrosted and analyzed independently. The PFU method was performed according to the Brazilian Pharmacopoeia. The samples (reconstituted vials and their aliquots) were serially diluted and each dilution were inoculated into wells of a microplate containing Vero cells and incubated at $37^{\circ} \mathrm{C} / 1 \mathrm{~h}$. The inoculum was aspirated and carboxymethylcellulose medium $3 \%$ was added and the plates were incubated at $38^{\circ} \mathrm{C} / 7$ days. The plates were fixed with $2 \%$ formaldehyde solution and stained with $1 \%$ violet crystal. The plaques presented were counted and the results were converted to $\log 10$ International Units /Human Dose (IU/HD). Statistical analysis of the results was performed by linear regression following ISO/GUIDE 35:2017.

Results: The reconstituted $\mathrm{RM}$ was not sufficiently stable at $(5 \pm 3)^{\circ} \mathrm{C}$ in both study models. But when aliquoted and stored at $(-20 \pm 10)^{\circ} \mathrm{C}$ it was considerate sufficiently stable for eight days with angular coefficient of $-0.014(\mathrm{LIC}=-0.045 ; \mathrm{LSC}=0,016)$.

Conclusion: It was concluded that the reconstitute YFV batch can be used as an RM in routine analysis if divided in aliquots and stored at $(-20 \pm 10)^{\circ} \mathrm{C}$ for 8 days since it has its property value established and it is stable. The used of aliquot after reconstitution can represent an economy of approximately 300 vials/year.

Keywords: yellow fever vaccine; reference material; potency assay 